\title{
Association between DSM-IV Anxiety Disorders and Suicidal Behaviors in a Community Sample of South Korean Adults
}

\author{
Seong-Jin Cho', Jin Pyo Hong' ${ }^{2}$, Jun-Young Lee ${ }^{3}$, Jeong Soo $\mathrm{Im}^{4}$, \\ Kyoung-Sae $\mathrm{Na}^{1}$, Jee Eun Park ${ }^{5}$, and Maeng Je $\mathrm{Cho}^{5} \bowtie$ \\ ${ }^{1}$ Department of Psychiatry, Gachon University Gil Medical Center, Incheon, Republic of Korea \\ 2Department of Psychiatry, Samsung Medical Center, Sungkyunkwan University College of Medicine, Seoul, Republic of Korea \\ ${ }^{3}$ Department of Psychiatry and Behavioral Science, College of Medicine, Seoul National University, Boramae Hospital, Seoul, Republic of Korea \\ ${ }^{4}$ Department of Preventive Medicine, Gachon University Gil Medical Center, Incheon, Republic of Korea \\ ${ }^{5}$ Department of Psychiatry and Behavioral Science, College of Medicine, Seoul National University, Seoul, Republic of Korea
}

\begin{abstract}
Objective There are conflicting reports about whether individual anxiety disorders are independently associated with suicidal ideation and suicide attempts. We examined whether anxiety disorders are related to suicidal ideation and suicide attempts in a community sample of South Korean adults.

Methods In-person interviews based on the South Korean version of the Composite International Diagnostic Interview were conducted to diagnose mental disorders. Multivariate logistic regression analysis was performed to examine whether anxiety disorders were associated with suicidal ideation and suicide attempts. Each regression model treated lifetime diagnosis of anxiety disorders as independent variables. Sociodemographic characteristics, cohabitation status, lifetime history of mood disorders, alcohol use disorders, and psychotic disorders were included as covariates.
\end{abstract}

Results Nationally representative sample of 6,510 South Korean adults aged 18-64 years was recruited. Multivariate analysis adjusted for psychiatric comorbidity and sociodemographic variables revealed that generalized anxiety disorder (GAD) [2.34, 95\% confidence interval (CI) $(1.27,4.33)$ ], post-traumatic stress disorder (PTSD) $(3.50,95 \%$ CI: 2.16, 5.68), specific phobia $(1.55,95 \%$ CI: 1.14, 2.11), social phobia (2.97, 95\% CI: 1.27, 6.94), and obsessive-compulsive disorder (OCD) (5.58, 95\% CI: 2.70, 11.6) were associated with suicidal ideation, whereas only social phobia $(3.78,95 \%$ CI: $1.41,10.1)$ and PTSD $(5.13,95 \%$ CI: 2.81 , 9.37) were associated with suicide attempts.

Conclusion Individual anxiety disorders are independently associated with suicidal ideation and/or suicide attempts. The importance of the early detection of anxiety disorders and of assessing the suicide risk in individuals with anxiety disorders is emphasized.

Psychiatry Investig 2016;13(6):595-600

Key Words Panic disorder, Generalized anxiety disorder, Obsessive-compulsive disorder, Post-traumatic stress disorder, Suicide.

\section{INTRODUCTION}

The suicide rate in South Korea increased to 31.2 per 100,000 persons in 2008 from 15 per 100,000 persons in 1999. ${ }^{1,2}$ Thus, public concerns about suicide risk and prevention have increased.

\footnotetext{
Received: May 18, 2015 Revised: July 5, 2015

Accepted: July 13, 2015 Available online: November 21, 2016

$\triangle$ Correspondence: Maeng Je Cho, MD, PhD

Department of Psychiatry and Behavioral Science, Seoul National University College of Medicine, 101 Daehak-ro, Jongno-gu, Seoul 03080, Republic of Korea

Tel: +82-2-2072-3155, Fax: +82-2-744-7241, E-mail: mjcho@snu.ac.kr

(a) This is an Open Access article distributed under the terms of the Creative Commons Attribution Non-Commercial License (http://creativecommons.org/licenses/bync/3.0) which permits unrestricted non-commercial use, distribution, and reproduction in any medium, provided the original work is properly cited.
}

It has been reported that suicide is strongly associated with suicidal ideation and attempts, ${ }^{3}$ and mood disorders, substance disorders, and schizophrenia have been established as risk factors for suicidal behaviors. ${ }^{4-7}$

Weissman et al. ${ }^{8}$ published a seminal article suggesting that panic disorder had a stronger association with suicide attempts compared to the general population ( $\mathrm{OR}=17.99,95 \% \mathrm{CI}$ : 12.18-26.58) based on more than 18,000 adults living in the community. Subsequently, many studies reported that anxiety disorders, including panic disorder, were associated with suicide ideation and suicide attempts, ${ }^{9,10}$ although there were inconsistent results and controversies. ${ }^{11}$ An overactivate hypothalamus-pituitary-adrenal HPA axis coupled with anxiety is one of the contributing factors for suicide. ${ }^{12}$ However, anx- 
iety disorder and related neuroendocrinological dysregulation alone might not directly cause the completed suicide. It has been suggested that comorbidity should be considered when evaluating risk factors for suicidal behaviors because psychiatric disorders can occur concurrently. ${ }^{13}$ Sareen ${ }^{14}$ argued that the controversy regarding associations between anxiety disorders and suicidal behaviors may be attributable to differences in the methods used to adjust for comorbid non-anxiety mental disorders. Accordingly, statistical analyses should control for each mental disorder separately.

To date, few studies using nationally representative community samples have examined whether individual anxiety disorders are associated with suicidal behavior after adjusting for other mental disorders.

Therefore, we aimed to examine the association between lifetime histories of individual anxiety disorders and lifetime histories of suicidal behavior while adjusting for sociodemographic factors, mood disorders, alcohol use disorders, and psychotic disorders.

\section{METHODS}

\section{Participants}

Data for this study were obtained from The Korean Epidemiologic Catchment Area Study-Replication (KECA-R), ${ }^{15}$ which was based on a stratified multistage area probability sample of the non-institutionalized South Korean population aged 1864 years. The response rate for the study was $81.7 \%$. The Institutional Review Board of the Seoul National University College of Medicine approved this study. All subjects were fully informed about the aims and methods of the study before the interview was conducted. Informed consent was obtained prior to participation. Detailed descriptions of the sampling procedures have been reported previously. ${ }^{15}$

Sociodemographic information including sex, age, cohabitation status, and education was obtained at the time of enrolment.

\section{Assessment of lifetime DSM-IV psychiatric disorders}

Each participant was assessed in person by a trained interviewer based on the Korean version of the Composite International Diagnostic Interview (K-CIDI), ${ }^{16}$ a fully structured, lay-administered diagnostic interview designed to identify psychiatric diagnoses according DSM-IV criteria. ${ }^{17}$ The KCIDI was previously validated by Cho et al. ${ }^{18}$ according to the World Health Organization guidelines. ${ }^{19}$ This study assessed the lifetime prevalence of the following DSM-IV psychiatric diagnoses using the K-CIDI: agoraphobia without panic disorder, generalized anxiety disorder (GAD), panic disorder without agoraphobia, post-traumatic stress disorder (PTSD), specific phobia, social phobia, obsessive-compulsive disorder (OCD), major depressive disorder, dysthymia, bipolar disorder, alcohol abuse or dependence, and psychotic disorders.

\section{Lifetime suicidal ideation, plan, and suicide attempts}

Respondents' histories of suicidal ideation and suicide attempts were assessed during the life-event history section of the interview based on the following questions. The suicidal ideation question was "Have you ever seriously thought about committing suicide?". The suicidal plan was measured by a question "Have you ever concretely planned suicide?" Suicidal attempt was measured by the following question, "Have you ever attempted suicide?" We further used following questions to differentiate suicidal attempt from suicidal gesture or selfharming behaviors: 1) I made a serious attempt to kill myself and it was only luck that I did not succeed. 2) I tried to kill myself but I knew the method was not fool-proof. 3) My attempt was a cry for help. I did not want to die. According to the previous studies, ${ }^{20,21}$ we only defined 1) and 2) as suicide attempt. Responding to 3) was defined as a suicidal gesture. Respondents who answered in the affirmative with regard to suicidal ideation $(n=1005)$, plan $(n=225)$, or suicide attempts $(n=166)$ were included in the analysis.

\section{Statistical analysis}

Cohabitation status was divided into two categories: cohabitation and living alone. Age and education were treated as continuous variables. Univariate logistic regression analyses were used to examine the relationship of lifetime suicidal ideation and suicide attempts with demographic variables and lifetime DSM-IV psychiatric diagnoses, including anxiety disorders. Next, multiple logistic regression analyses were performed using variables found to be associated with suicidal ideation, suicidal plan, suicide attempts, and major depressive disorder (MDD) in the univariate logistic regression analyses. The multiple logistic regression analyses treated lifetime diagnoses of anxiety disorders (agoraphobia, GAD, panic disorder, PTSD, specific phobia, social phobia, and OCD) as independent variables. Covariates in the analysis were sex, age, education, cohabitation status, lifetime diagnosis of MDD, dysthymia, bipolar disorder, alcohol abuse or dependence, and psychotic disorders. When the MDD was set as a dependent variable, covariates were sex, age, education, cohabitation status, lifetime diagnosis of dysthymia, bipolar disorder, alcohol abuse or dependence, and psychotic disorders. Adjusted odds ratios (ORs) and 95\% CIs were calculated based on these multiple logistic regression models. Statistical analyses were performed using SPSS software, version 16.0. 


\section{RESULTS}

Table 1 presents the results of the univariate analysis of the association of lifetime suicidal ideation and suicide attempts with demographic variables. Being female ( $\mathrm{OR}=1.39,95 \% \mathrm{CI}$ : 1.21-1.61; $\mathrm{OR}=1.45,95 \% \mathrm{CI}: 1.04-2.02)$ and living alone $(\mathrm{OR}=$ 1.29, 95\% CI: $1.12-1.49$; OR=1.83, 95\% CI: 1.34-2.50) were significantly associated with both suicidal ideation and suicide attempts. Associations of demographic variables, such as age and education, with suicidal ideation and suicide attempts were not significant with the exception of the correlation between education and suicide attempts $(\mathrm{OR}=0.92,95 \% \mathrm{CI}$ : 0.89-0.97).

Table 2 presents the relationship of each psychiatric diag- nosis with suicidal ideation and suicide attempts. All psychiatric disorders were significantly associated with suicidal ideation, and, with the exceptions of dysthymia (OR=2.31, 95\% CI: 0.55-9.71) and agoraphobia without panic disorder $(\mathrm{OR}=$ 3.17, 95\% CI: 0.41-24.5), with suicide attempts.

Table 3 presents the relationship of anxiety disorders with suicidal ideation and suicide attempts by psychiatric comorbidity and sociodemographic characteristics. GAD (OR= 2.34, 95\% CI: 1.27-4.33), PTSD (OR=3.50, 95\% CI: 2.165.68), specific phobia (OR=1.55, 95\% CI: $1.14-2.11)$, social phobia (OR=2.97, 95\% CI: 1.27-6.94), and OCD (OR=5.58, 95\% CI: 2.70-11.6) remained significantly associated with suicidal ideation. Agoraphobia without panic disorder $(\mathrm{OR}=$ 3.12, 95\% CI: 0.94-10.3) and panic disorder without agora-

Table 1. Association of demographic variables with suicidal ideation and suicide attempts

\begin{tabular}{|c|c|c|c|c|c|c|}
\hline & \multicolumn{2}{|c|}{ Suicidal ideation } & \multicolumn{2}{|c|}{ Suicidal plan } & \multicolumn{2}{|c|}{ Suicide attempt } \\
\hline & Proportion & OR (95\% CI) & Proportion & OR (95\% CI) & Proportion & OR (95\% CI) \\
\hline Total sample & $15.4 \%(1005 / 6454)$ & & $3.42 \%(225 / 6587)$ & & $2.6 \%(166 / 6445)$ & \\
\hline Sex (Female) & $17.2 \%(672 / 3896)$ & $1.39(1.21,1.61)^{\dagger}$ & $4.3 \%(169 / 3893)$ & $2.03(1.49,2.75)^{\ddagger}$ & $3.9 \%(114 / 3888)$ & $1.45(1.04,2.02)^{*}$ \\
\hline \multirow{2}{*}{$\begin{array}{l}\text { Cohabitation status } \\
\text { (Not cohabiting) }\end{array}$} & $18.0 \%(341 / 1894)$ & $1.29(1.12,1.49)^{\dagger}$ & $4.7 \%(89 / 1892)$ & $1.60(1.22,2.12)^{\dagger}$ & $4.7 \%(71 / 1894)$ & $1.83(1.34,2.50)^{\ddagger}$ \\
\hline & Mean (SD) & OR (95\% CI) & Mean (SD) & OR $(95 \% \mathrm{CI})$ & Mean (SD) & OR $(95 \% \mathrm{CI})$ \\
\hline Age (years) & $40.8(11.8)$ & $1.00(0.99,1.00)$ & $40.9(11.8)$ & $1.00(0.99,1.01)$ & $41.4(11.7)$ & $1.00(0.99,1.02)$ \\
\hline Education (years) & $12.2(3.56)$ & $0.99(0.97,1.01)$ & $11.8(3.9)$ & $0.97(0.93,1.00)$ & $11.3(4.2)$ & $0.92(0.89,0.97)^{\dagger}$ \\
\hline
\end{tabular}

${ }^{*}<0.05,{ }^{\dagger}<0.01,{ }^{\ddagger}<0.001$

Table 2. Univariate logistic regression analysis of individual anxiety disorders on lifetime suicidal ideation and suicide attempt

\begin{tabular}{|c|c|c|c|c|c|c|}
\hline & \multicolumn{2}{|c|}{ Suicidal ideation } & \multicolumn{2}{|c|}{ Suicidal plan } & \multicolumn{2}{|c|}{ Suicide attempt } \\
\hline & Proportion & OR $(95 \% \mathrm{CI})$ & Proportion & OR $(95 \% \mathrm{CI})$ & Proportion & OR (95\% CI) \\
\hline $\begin{array}{l}\text { Alcohol abuse or } \\
\text { dependence }\end{array}$ & $23.3 \%(206 / 886)$ & $1.81(1.52,2.15)^{\ddagger}$ & $6.2 \%(55 / 886)$ & $2.21(1.54,2.87)^{\ddagger}$ & $5.5 \%(49 / 886)$ & $2.73(1.94,3.84)^{\ddagger}$ \\
\hline $\begin{array}{l}\text { Major depressive } \\
\text { disorder }\end{array}$ & $51.2 \%(199 / 389)$ & $6.83(5.53,8.45)^{\ddagger}$ & $18.0 \%(70 / 389)$ & $8.39(6.19,11.4)^{\ddagger}$ & $13.1 \%(51 / 389)$ & $7.81(5.52,11.1)^{\ddagger}$ \\
\hline Dysthymia & $42.9 \%(15 / 35)$ & $4.11(2.10,8.06)^{\ddagger}$ & $2.9 \%(1 / 35)$ & $0.81(0.11,5.97)$ & $2.9 \%(1 / 35)$ & $2.31(0.55,9.71)$ \\
\hline Bipolar disorder & $72.2 \%(13 / 18)$ & $14.3(5.08,40.1)^{\dagger}$ & $16.7 \%(3 / 18)$ & $34.2(13.0,89.4)^{\dagger}$ & $44.4 \%(8 / 18)$ & $31.8(12.4,81.6)^{\ddagger}$ \\
\hline Psychotic disorder & $32.1 \%(9 / 28)$ & $2.58(1.17,5.72)^{*}$ & $14.3 \%(4 / 28)$ & $4.68(1.61,13.6)^{\dagger}$ & $10.7 \%(3 / 28)$ & $4.61(1.38,15.4)^{*}$ \\
\hline $\begin{array}{l}\text { Agoraphobia without } \\
\text { panic disorder }\end{array}$ & $46.2 \%(6 / 13)$ & $4.67(1.57,13.9)^{\dagger}$ & $46.2 \%(6 / 13)$ & $2.31(1.57,13.9)^{\dagger}$ & $7.7 \%(1 / 13)$ & $3.17(0.41,24.5)$ \\
\hline $\begin{array}{l}\text { Generalized anxiety } \\
\text { disorder }\end{array}$ & $51.9 \%(27 / 52)$ & $5.99(3.46,10.4)^{\dagger}$ & $26.9 \%(14 / 52)$ & $10.81(5.76,20.2)^{\ddagger}$ & $15.4 \%(8 / 52)$ & $10.25(3.78,27.8)^{\ddagger}$ \\
\hline $\begin{array}{l}\text { Panic disorder } \\
\text { without agoraphobia }\end{array}$ & $50 \%(6 / 12)$ & $5.45(1.75,16.9)^{\dagger}$ & $16.7 \%(2 / 12)$ & $5.57(1.21,25.6)^{*}$ & $16.7 \%(2 / 12)$ & $7.66(1.67,35.2)^{\dagger}$ \\
\hline $\begin{array}{l}\text { Post-traumatic stress } \\
\text { disorder }\end{array}$ & $58.0 \%(51 / 88)$ & $7.82(5.09,12.0)^{\ddagger}$ & $27.3 \%(24 / 88)$ & $11.49(7.04,18.8)^{\ddagger}$ & $25.0 \%(22 / 88)$ & $14.4(8.6,24.0)^{\ddagger}$ \\
\hline Specific phobia & $29.4 \%(78 / 265)$ & $2.37(1.80,3.11)^{\ddagger}$ & $9.8 \%(26 / 265)$ & $3.29(2.14,5.05)^{\ddagger}$ & $6.4 \%(17 / 264)$ & $2.78(1.66,4.66)^{\ddagger}$ \\
\hline Social phobia & $61.3 \%(19 / 31)$ & $8.73(4.23,18.0)^{\ddagger}$ & $32.3 \%(10 / 31)$ & $13.74(6.4,29.5)^{\ddagger}$ & $29.0 \%(9 / 31)$ & $16.3(7.40,36.0)^{\ddagger}$ \\
\hline $\begin{array}{l}\text { Obsessive-compulsive } \\
\text { disorder }\end{array}$ & $65.0 \%(26 / 40)$ & $10.3(5.37,19.8)^{\ddagger}$ & $25.0 \%(10 / 40)$ & $9.6(4.6,19.9)^{\ddagger}$ & $20.0 \%(8 / 40)$ & $9.9(4.5,21.8)^{\ddagger}$ \\
\hline
\end{tabular}


Table 3. Multivariate logistic regression analysis of lifetime suicidal ideation and suicide attempts adjusted for psychiatric comorbidities and demographic variables

\begin{tabular}{|c|c|c|c|}
\hline & Suicidal ideation & Suicide plan & Suicidal attempt \\
\hline & Adjusted OR (95\% CI) & Adjusted OR (95\% CI) & Adjusted OR (95\% CI) \\
\hline Agoraphobia without panic disorder & $3.12(0.94,10.3)$ & $1.16(0.13,10.1)$ & $1.17(0.13,10.7)$ \\
\hline Generalized anxiety disorder & $2.34(1.27,4.33)^{\dagger}$ & $3.61(1.72,7.53)^{\dagger}$ & $2.07(0.81,5.27)$ \\
\hline Panic disorder without agoraphobia & $2.34(0.68,8.09)$ & $2.09(0.40,10.8)$ & $2.77(0.53,14.4)$ \\
\hline Post-traumatic stress disorder & $3.50(2.16,5.68)^{\ddagger}$ & $4.04(2.27,7.21)^{\ddagger}$ & $5.13(2.81,9.37)^{\ddagger}$ \\
\hline Specific phobia & $1.55(1.14,2.11)^{\dagger}$ & $1.57(0.95,2.59)$ & $1.28(0.69,2.37)$ \\
\hline Social phobia & $2.97(1.27,6.94)^{*}$ & $3.67(1.41,9.50)^{\dagger}$ & $3.78(1.41,10.1)^{\dagger}$ \\
\hline Obsessive compulsive disorder & $5.58(2.70,11.6)^{\ddagger}$ & $2.82(1.08,7.40)^{*}$ & $2.36(0.79,7.04)$ \\
\hline
\end{tabular}

Adjusted for sex, age, cohabitation status, education, alcohol abuse or dependence, major depressive disorder, dysthymia, bipolar disorder, and psychotic disorder using unweighted data. ${ }^{*}<0.05,{ }^{\dagger}<0.01, \ddagger<0.001$

Table 4. Multivariate logistic regression analysis of lifetime major depressive disorder adjusted for psychiatric comorbidities and demographic variables

\begin{tabular}{lrc}
\hline & $\mathrm{p}$ & $\begin{array}{c}\text { Adjusted OR } \\
(95 \% \mathrm{CI})\end{array}$ \\
\hline Agoraphobia without panic disorder & 0.424 & $2.09(0.34,12.7)$ \\
Generalized anxiety disorder & $<0.001$ & $10.24(5.59,18.75)$ \\
Panic disorder without agoraphobia & 0.001 & $7.82(2.31,26.5)$ \\
Post-traumatic stress disorder & $<0.001$ & $4.48(2.69,7.48)$ \\
Specific phobia & $<0.001$ & $2.53(1.75,3.68)$ \\
Social phobia & $<0.001$ & $7.00(3.03,16.16)$ \\
Obsessive-compulsive disorder & 0.004 & $3.53(1.50,8.32)$ \\
\hline
\end{tabular}

Adjusted for sex, age, cohabitation status, education, alcohol abuse or dependence, dysthymia, bipolar disorder, and psychotic disorder using unweighted data

phobia (OR=2.34, 95\% CI: 0.68-8.09) were no longer significantly associated with suicidal ideation. Social phobia (OR= 3.78, 95\% CI: $1.41-10.1)$ and PTSD (OR=5.13, 95\% CI: 2.819.37) remained significantly associated with suicide attempts, whereas panic disorder without agoraphobia $(\mathrm{OR}=2.77,95 \%$ CI: 0.53-14.4), specific phobia (OR=1.28, 95\% CI: 0.69-2.37), GAD (OR=2.07, 95\% CI: 0.81-5.27), and OCD (OR=2.36, 95\% CI: 0.79-7.04) did not.

Table 4 presented the relationship of anxiety disorders with MDD. Among anxiety disorders, GAD (OR=10.24, 95\% CI: 5.59-18.75) had the highest association with MDD, followed by panic disorder (OR=7.82, 95\% CI: 2.31-26.55), social phobia (OR=7.00, 95\% CI:3.03-16.16), and PTSD (OR=4.48, 95\% CI: 2.69-7.48). Agoraphobia (OR=2.09, 95\% CI: 0.34-12.73) was the only anxiety disorder which was had no association with MDD.

\section{DISCUSSION}

This is the first epidemiological study to investigate the rela- tionship between DSM-IV anxiety disorders and suicidal behavior using a nationally representative South Korean sample.

The findings of this study suggest that GAD, PTSD, social phobia, specific phobia, and OCD are independent risk factors for suicidal ideation, whereas only PTSD and social phobia are predictors of suicide attempts. Panic disorder and agoraphobia were not associated with suicidal ideation, plan, and attempt. These results were obtained after adjusting for other important variables, including major DSM-IV axis I disorders and sociodemographic variables including age, sex, cohabitation status, and education.

Particularly, statistical significance of the influence of PTSD increased with the degree of suicidality. Adjusted OR of PTSD for suicidal idea, suicidal plan, and suicidal attempt were 3.50, 4.04 , and 5.13, respectively. These results suggested that the proclivity of PTSD for suicide is the severest among anxiety disorders. Several studies have consistently reported that patients with PTSD have high rates of suicidality. ${ }^{22}$ Our findings were similar to previous studies, in which PTSD was commonly correlated with suicidal attempts. ${ }^{23,24}$ Although the impact of individual anxiety disorders for suicide risk has been addressed by previous studies, many studies focused on PTSD alone, or other anxiety disorders excluding PTSD. ${ }^{25}$ However, our study measured all sort of anxiety disorders, which is the principal difference with the previous studies.

There have been inconsistent results concerning social phobia. Whereas we showed that social phobia is associated with suicidal ideation, plan, and attempt, Schneider et al. ${ }^{26}$ found that social phobia was associated with suicidal ideation but not with suicide attempts. These differences may be attributable, in part, to the fact that that study included variables coded for three and more lifetime DSM-III-R disorders and included antisocial personality disorder in the multivariate logistic regression analysis, which may have reduced the significance of some predictors of suicidal behaviors.

GAD and OCD were associated with suicidal ideation and 
plan, but not with suicidal attempts. Previous studies have consistently found that patients with GAD, one of the most common mental illnesses, with a lifetime prevalence of more than $5 \%,{ }^{27}$ had higher suicidality than did those without $\mathrm{GAD}^{28,29}$ A previous Chinese study reported that $37.6 \%$ of patients with a lifetime history of GAD had a history of suicide attempts, whereas only $4.2 \%$ of those without GAD had such a history. ${ }^{29} \mathrm{~A}$ study conducted in Europe also reported that suicide attempts and suicidal ideation were more frequent in patients with GAD than in those without. ${ }^{28}$ Regarding suicidal risk of OCD, Sareen et al. ${ }^{30}$ found that social phobia and OCD were associated with suicidal ideation but not with suicide attempts. However, the Epidemiological Catchment Area Study found that OCD with or without a lifetime history of any other psychiatric disorder was associated with suicide attempts. ${ }^{31}$ Possible reason for the differential association between anxiety disorders (GAD and OCD) and suicidal behaviors (suicidal idea and suicide attempt) may result from the comorbid psychiatric disorders. Sareen et al. ${ }^{30}$ proposed that these anxiety disorders may have different relationships with suicidal behavior; that is, suicidal ideation may be directly related to social phobia and OCD, whereas suicidal attempt may be indirectly related and mediated by comorbid psychiatric disorders (depression, substance abuse, etc.). Since our study controlled possible influences of comorbid psychiatric disorders, thereby blocking the indirect pathway, GAD and OCD were not associated with suicide attempt. In OCD, clinical symptomatology might have influences on the suicidal behavior. For example, Kamath et al. ${ }^{32}$ reported that religion, reassurance seeking, and the repetitive symptoms of OCD were associated with suicide attempts.

Results from the current study differed from the results of previous studies that found panic disorder to be associated with suicidal ideation and suicide attempts. ${ }^{8,11,23}$ The association of panic disorder with suicidality has been a recent focus, since it is assumed likely that patients with panic disorder have a fear of death, which would prevent them from committing suicide. Although comorbidity of panic disorder may play an important role for suicidal risk, there have been inconsistent conclusions on whether panic disorder independently increased suicidality. ${ }^{23,33}$ Whereas panic disorder had no association with suicidality, it showed a close relationship with MDD. Following GAD, panic disorder was the second highest impacting anxiety disorder for the prevalence of MDD. Such observations were suggestive of different mechanisms of influence by anxiety disorder on suicide vs. depression. These considerations, which suggesting that comorbid psychiatric disorders have crucial role in the suicide attempt in anxiety disorders, may be applicable to the association between suicidal behaviors and specific phobia, in which only suicide idea was asso- ciated with specific phobia.

Our study has several notable limitations. First, we could not examine causal relationships between DSM-IV anxiety disorders and suicidal behavior, as this study used a crosssectional design. Second, we assessed suicidality based solely on subjects' responses to the questionnaire and did not obtain confirmatory information. Third, because a number of psychiatric disorders have low 1-year prevalence, only lifetime prevalence rates were examined, which could have increased recall bias and further confused the causal relationships between suicidality and anxiety disorders. It is noteworthy that the prevalence of most of the DSM-IV psychiatric disorders were lower than those reported in other regions, despite the use of lifetime prevalence of anxiety disorders. For example, the prevalence rate of panic disorder without agoraphobia $(0.19 \%)$ in South Korea was lower than the prevalence rate $3.7 \%$ in the United States. ${ }^{34}$ However, the suicide rate has been the highest in South Korea compared to other OECD countries. ${ }^{35}$ We surmised that the discrepancy between low psychiatric disorders and high suicide rates might be due to sociocultural factors such as stigma and prejudice. The fear of stigma might hinder the mentally ill from disclosing their psychiatric symptoms and seeking medical help. Several published findings have revealed the importance of socio-cultural factors, without a direct investigation on the relationship among stigma, psychiatric disorders, and suicide in South Korea. It has been reported that people living in regions with low suicide rates had less stigma associated with mental illness than regions with high suicide rates. ${ }^{36}$ Another study suggested that suicide rates were twice as high among South Korean emigrants in Japan than non-emigrants, indicative of a strong association with prejudice and economic factors. ${ }^{37}$

In conclusion, our study revealed that anxiety disorders, except for panic disorder and agoraphobia, were associated with suicidal ideation and plan. PTSD was the most influential factor for suicide attempt on adjusting for sociodemographic variables and other anxiety disorders. Future studies are necessary to identify psychosocial and neurobiological mechanisms underlying the suicidality of anxiety disorders. Cumulative effects of prior suicide attempts, comorbidity, and clinical characteristics of individual anxiety disorders should also be considered.

\section{REFERENCES}

1. Statistics Korea. Annual Report on the Cause of Death Statistics. Daejeon: Statistics Korea; 1999.

2. Statistics Korea. Annual Report on the Cause of Death Statistics. Daejeon: Statistics Korea; 2008.

3. Suominen K, Isometsa E, Suokas J, Haukka J, Achte K, Lonnqvist J. Completed suicide after a suicide attempt: a 37-year follow-up study. Am J Psychiatry 2004;161:562-563. 
4. Henriksson MM, Aro HM, Marttunen MJ, Heikkinen ME, Isometsa ET, Kuoppasalmi KI, et al. Mental disorders and comorbidity in suicide. Am J Psychiatry 1993;150:935-940.

5. ten Have M, Vollebergh W, Bijl R, Nolen WA. Bipolar disorder in the general population in The Netherlands (prevalence, consequences and care utilisation): results from The Netherlands Mental Health Survey and Incidence Study (NEMESIS). J Affect Disord 2002;68:203-213.

6. Verona E, Sachs-Ericsson N, Joiner TE Jr. Suicide attempts associated with externalizing psychopathology in an epidemiological sample. Am J Psychiatry 2004;161:444-451.

7. Phillips MR, Yang G, Li S, Li Y. Suicide and the unique prevalence pattern of schizophrenia in mainland China: a retrospective observational study. Lancet 2004;364:1062-1068.

8. Weissman MM, Klerman GL, Markowitz JS, Ouellette R. Suicidal ideation and suicide attempts in panic disorder and attacks. N Engl J Med 1989;321:1209-1214.

9. Nock MK, Borges G, Bromet EJ, Alonso J, Angermeyer M, Beautrais A, et al. Cross-national prevalence and risk factors for suicidal ideation, plans and attempts. Br J Psychiatry 2008;192:98-105.

10. Bolton JM, Cox BJ, Afifi TO, Enns MW, Bienvenu OJ, Sareen J. Anxiety disorders and risk for suicide attempts: findings from the Baltimore Epidemiologic Catchment area follow-up study. Depress Anxiety 2008; 25:477-481.

11. Goodwin RD, Roy-Byrne P. Panic and suicidal ideation and suicide attempts: results from the National Comorbidity Survey. Depress Anxiety 2006;23:124-132.

12. Turecki G. The molecular bases of the suicidal brain. Nat Rev Neurosci 2014;15:802-816.

13. Nock MK, Hwang I, Sampson NA, Kessler RC. Mental disorders, comorbidity and suicidal behavior: results from the National Comorbidity Survey Replication. Mol Psychiatry 2010;15:868-876.

14. Sareen J. Anxiety disorders and risk for suicide: why such controversy? Depress Anxiety 2011;28:941-945.

15. Cho SJ, Hong JP, Hahm BJ, Jeon HJ, Chang SM, Cho MJ, et al. Restless legs syndrome in a community sample of Korean adults: prevalence, impact on quality of life, and association with DSM-IV psychiatric disorders. Sleep 2009;32:1069-1076.

16. World Health Organization. Composite International Diagnostic Interview (CIDI) Version 1.0. Geneva: World Health Organization; 1990.

17. APA. Diagnostic and Statistical Manual of Mental Disorders. Washington DC American Psychiatric Association (APA); 1994.

18. Cho MJ, Hahm BJ, Suh DW, Hong JP, Bae JN, Kim JK, et al. Development of a Korea Version of the Composite International Diagnostic Interview (K-CIDI). J Korean Neuropsychiatr Assoc 2002;41:123-137.

19. World Health Organization. Procedures for the Development of New Language Version of the WHO Composite International Diagnostic Interview (WHO-CIDI). Geneva: World Health Organization; 1997.

20. Nock MK, Kessler RC. Prevalence of and risk factors for suicide attempts versus suicide gestures: analysis of the National Comorbidity Survey. J Abnorm Psychol 2006;115:616-623.

21. Borges G, Angst J, Nock MK, Ruscio AM, Kessler RC. Risk factors for the incidence and persistence of suicide-related outcomes: a 10-year fol- low-up study using the National Comorbidity Surveys. J Affect Disord 2008;105:25-33.

22. Panagioti M, Gooding P, Tarrier N. Post-traumatic stress disorder and suicidal behavior: a narrative review. Clin Psychol Rev 2009;29:471-482.

23. Nepon J, Belik SL, Bolton J, Sareen J. The relationship between anxiety disorders and suicide attempts: findings from the National Epidemiologic Survey on Alcohol and Related Conditions. Depress Anxiety 2010; 27:791-798.

24. Cougle JR, Keough ME, Riccardi CJ, Sachs-Ericsson N. Anxiety disorders and suicidality in the National Comorbidity Survey-Replication. J Psychiatr Res 2009;43:825-829.

25. Kanwar A, Malik S, Prokop LJ, Sim LA, Feldstein D, Wang Z, et al. The association between anxiety disorders and suicidal behaviors: a systematic review and meta-analysis. Depress Anxiety 2013;30:917-929.

26. Schneier FR, Johnson J, Hornig CD, Liebowitz MR, Weissman MM. Social phobia. Comorbidity and morbidity in an epidemiologic sample. Arch Gen Psychiatry 1992;49:282-288.

27. Kessler RC, Berglund P, Demler O, Jin R, Merikangas KR, Walters EE. Lifetime prevalence and age-of-onset distributions of DSM-IV disorders in the National Comorbidity Survey Replication. Arch Gen Psychiatry 2005;62:593-602.

28. Bernal M, Haro JM, Bernert S, Brugha T, de Graaf R, Bruffaerts R, et al. Risk factors for suicidality in Europe: results from the ESEMED study. J Affect Disord 2007;101:27-34.

29. Ma X, Xiang YT, Cai ZJ, Lu JY, Li SR, Xiang YQ, et al. Generalized anxiety disorder in China: prevalence, sociodemographic correlates, comorbidity, and suicide attempts. Perspect Psychiatr Care 2009;45:119-127.

30. Sareen J, Cox BJ, Afifi TO, de Graaf R, Asmundson GJ, ten Have M, et al. Anxiety disorders and risk for suicidal ideation and suicide attempts: a population-based longitudinal study of adults. Arch Gen Psychiatry 2005;62:1249-1257.

31. Hollander E, Greenwald S, Neville D, Johnson J, Hornig CD, Weissman MM. Uncomplicated and comorbid obsessive-compulsive disorder in an epidemiologic sample. Depress Anxiety 1996-1997;4:111-119.

32. Kamath P, Reddy YC, Kandavel T. Suicidal behavior in obsessive-compulsive disorder. J Clin Psychiatry 2007;68:1741-1750.

33. Hornig CD, McNally RJ. Panic disorder and suicide attempt. A reanalysis of data from the Epidemiologic Catchment Area study. Br J Psychiatry 1995;167:76-79.

34. Kessler RC, Chiu WT, Jin R, Ruscio AM, Shear K, Walters EE. The epidemiology of panic attacks, panic disorder, and agoraphobia in the National Comorbidity Survey Replication. Arch Gen Psychiatry 2006; 63:415-424.

35. Statistics Korea. Annual Report on the Cause of Death Statistics. Daejeon: Statistics Korea; 2013.

36. Reynders A, Kerkhof AJ, Molenberghs G, Van Audenhove C. Attitudes and stigma in relation to help-seeking intentions for psychological problems in low and high suicide rate regions. Soc Psychiatry Psychiatr Epidemiol 2013;49:231-239.

37. Lester D, Saito Y, Ben Park BC. Suicide among foreign residents of Japan. Psychol Rep 2011;108:139-140. 